\title{
Correlation between nanoscale elasticity, semiconductivity, and structural order in functionalized polyaniline thin films \\ Supporting Information
}

Chen, Po-Yen ${ }^{1,2}$, Hung, Hua-Li ${ }^{2}$,Han, Chien-Chung ${ }^{2 *}$, and Chiu, Hsiang-Chih ${ }^{1 *}$

${ }^{1}$ Department of Physics, National Taiwan Normal University, Taipei, Taiwan, Republic of China

${ }^{2}$ Department of Chemistry, National Tsing Hua University, Hsinchu, Taiwan, Republic of China

\section{UV-vis spectrum results and a brief discussion:}

The UV-vis spectrum of the emeraldine base (EB) form of the original unsubstituted PANI shows a typical two-band spectral featuring (Figure S1(a)), with a $\pi-\pi^{*}$ transition band of the benzenoid ring appearing at $\sim 320 \mathrm{~nm}$ and an exciton band of the quinoid rings at $\sim 634 \mathrm{~nm} .{ }^{1-2}$ The butylthio-substituted polyaniline, PANI-SBu, was prepared via the CRS (concurrent reduction and substitution) method, ${ }^{3-8}$ by reacting an unsubstituted PANI(EB) with butane-1-thiol in $\mathrm{MeOH}$ under $\mathrm{N}_{2}$ atmosphere. ${ }^{6-7}$ After the quinoid ring reacts with butane-1-thiol, it transforms into a buylthio-substituted benzenoid ring. As the CRS reaction progress, the oxidation degree of the PANI backbone became lower accompanying with the intensity reduction of the exciton band (Figure S1(b)), while the number of the butylthio substituent increased. Since a PANI EB contains $\sim 25 \%$ of the quinoid ring, the resultant PANI-SBu chain would thus contain one butylthio group in every four repeating aniline units. ${ }^{6}$ As confirmed by the spectral change in Fig. 2S, the fully reduced leucoemeraldine base form of PANI-SBu (Figure S1(b)), after removing unreacted butane-1-thiol via purification, could be reoxidized gradually back to the most stable emeraldine base form (Figure S1(b)) by stirring it under air atmosphere in MeOH. Since the butylthio-substituted aniline repeat units have higher electron-density than their neighboring unsubstituted aniline repeat unit, ${ }^{9}$ they would be preferably oxidized back to the quinoid ring. To obtain the preferred EB form of PANI-SBu, the air-oxidation treatment should be stopped by removing the air, when the $\lambda_{\max }$ intensity ratio between the exciton band and the $\pi-\pi^{*}$ band reached about 0.6 0.8 .

(a)

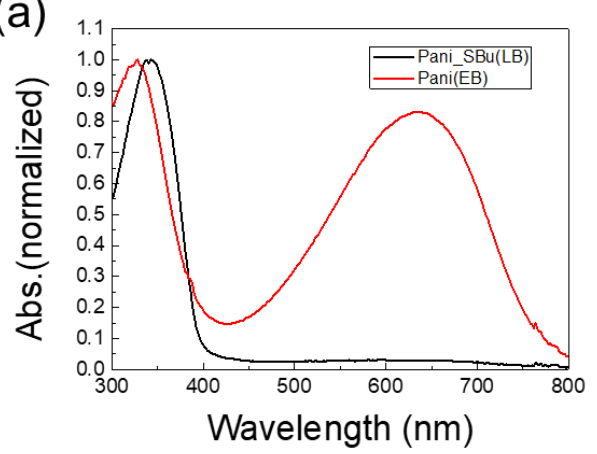

(b)

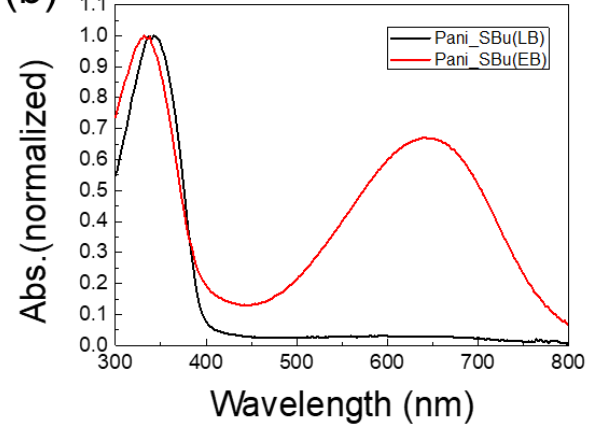

Figure S1. The UV absorption spectrum of PANI.

\section{The detailed synthesis procedure of PANI-SBu}

The powder of PANI(EB) (0.5 g) was stirred with $0.1 \mathrm{M}$ butane-1-thiol in methanol $(25 \mathrm{~mL})$ under nitrogen. The solution was taken out $1 \mathrm{~mL}$ every 2 hours and monitored with the UV-vis spectrum. The attachment of butylthio group to PANI was completed when the exciton band at $634 \mathrm{~nm}$ disappeared totally. The product was collected with vacuum filtration to get the PANI-SBu powder. The powder was soaked and stirred in the degassed methanol $(50 \mathrm{~mL})$ for $30 \mathrm{~min}$ and then collected with vacuum filtration to remove unreacted butane-1-thiol. Such cleaning treatment was repeated for 5 times. Finally the solid was dried in the oven under vacuum and $70^{\circ} \mathrm{C}$ for 3 hours. 


\section{Surface wettability of used substrates:}

The properties of the film such as thickness may be influenced by the shape of solution droplet cast on the substrates, depending on the interactions between NMP and the substrate surfaces. Because NMP is miscible with water at all temperatures, by measuring the water contact angle (WCA) on the substrates we used, we may get insights into how the surface wettability of the substrate may do to the PANI-SBu films. A more hydrophobic surface may lead to a thicker film, while a hydrophilic surface may result in a thinner one.

We tested the surface wettability of the used substrates by using a home-built goniometer. A water droplet of $2 \mu \mathrm{L}$ was placed on the surface of silicon, glass, and PEDOT:PSS-coated ITO to evaluate the WCA. The images of water droplet on these substrates are respectively shown in Figure S2 (a), (b). and (c). The WCA on silicon, glass and PEDOT:PSS-coated ITO substrates are respectively found to be $13.7 \pm 3.4,31.3 \pm 3.6$, and $34.7 \pm 6.6$, which indicate that all substrate surfaces were hydrophilic. Therefore, the droplet of PANI-SBu/NMP on these substrates should be very similar and so are the properties of the films.

(a) Silicon:

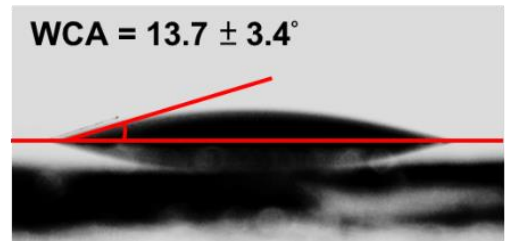

(b) Glass :

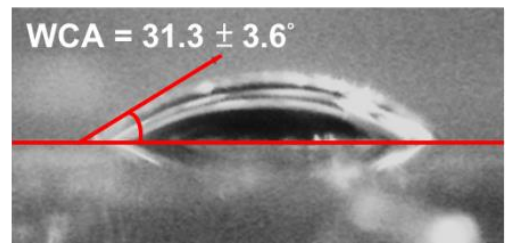

(c) PEDOT:PSS/ITO:

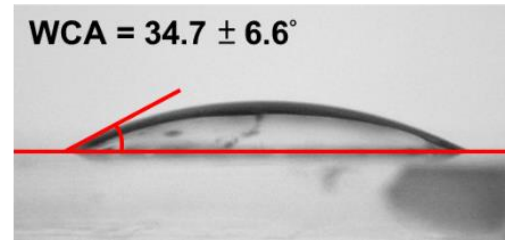

Figure S2. Water contact angle (WCA) measurements on (a) silicon; (b) glass; (c) PEDOT:PSS-coated ITO substrates. The shape of the water droplets indicates that all substrate surfaces are hydrophilic.

\section{Detailed PF-QNM calibration procedures:}

We first applied the PF-QNM on the PS-LDPE surface, a blend of polystyrene and polyolefin elastomer, whose elasticity is well known, to perform our system calibration. The elastic moduli of PS and LDPE are $2 \mathrm{GPa}$ and $0.1 \mathrm{GPa}$, respectively. During the calibration process, the force-indentation curve was obtained for every point of the scanned area, and the indentation depth (sample deformation) was kept at $\sim 2 \mathrm{~nm}$. The typical images from PS-LDPE surface during calibration are shown in Figure $\mathrm{S} 3$. The measured elasticity of the PS region is about $2 \mathrm{GPa}$. Then we used the same system to perform PF-QNM on PANI-SBu thin films.

(a)

(b)
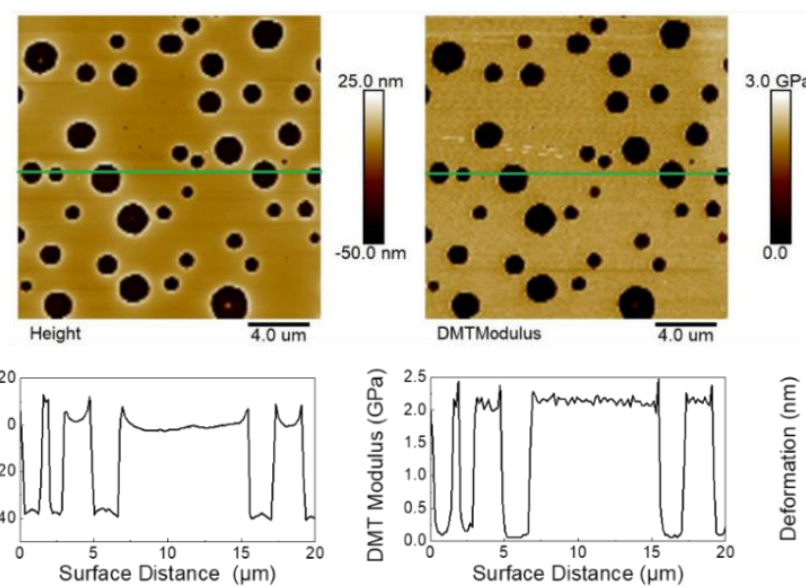
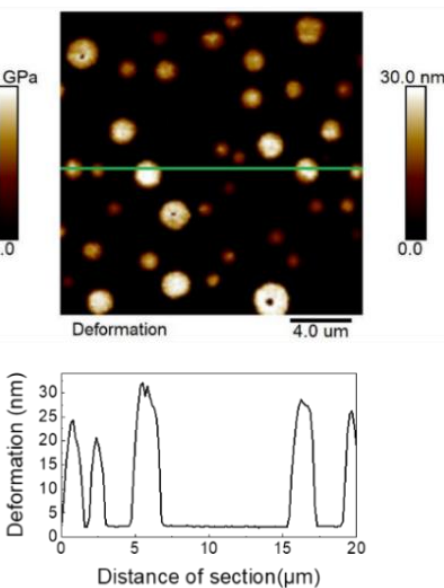
Figure S3. (a) Topography, elasticity, and deformation images obtained during the PF-QNM calibration procedure on PS-LDPE; (b) The variations of data along the green lines indicated in (a). The measured elastic modulus of PS region is $\sim 2 \mathrm{GPa}$ while the indentation depth is $\sim 2.2 \mathrm{~nm}$.

\section{The UV-vis absorption after stirring for long period of time:}
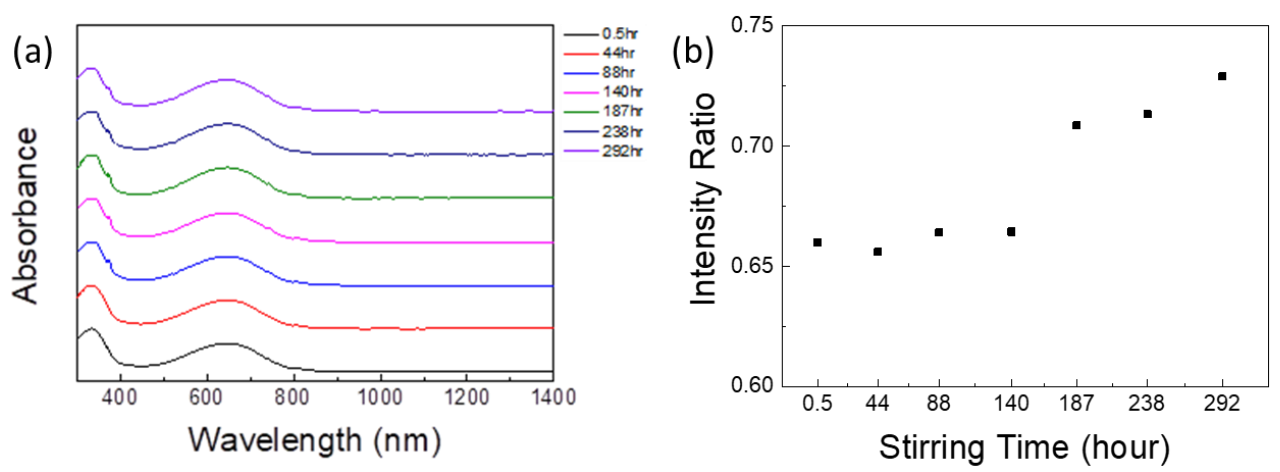

Figure S4. (a)The UV-vis spectrum results after stirring for long period of time (b)The intensity ratio between the exciton band (associated with diiminoquinoid ring structure) vs. the $\pi-\pi^{*}$ band (associated with the diaminobenzenoid ring structure) after stirring for long period of time.

\section{The measured elastic moduli vs. stirring time for all the films in PANI-SBu-1 and PANI-SBu2:}

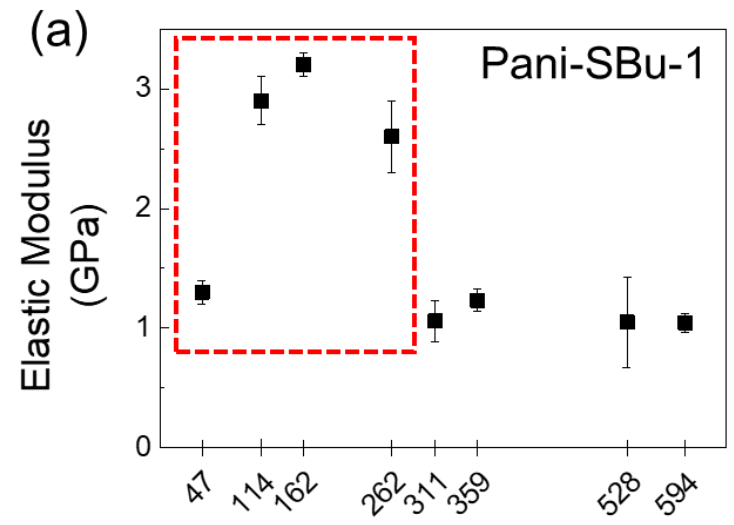

Stirring Time (hour)

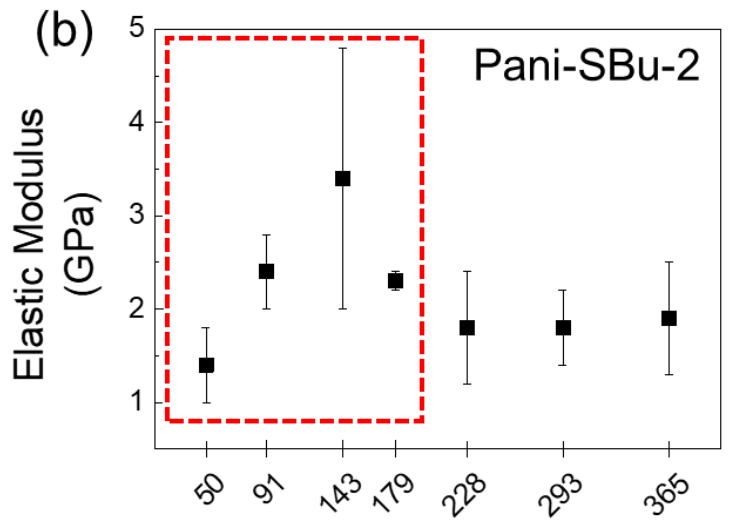

Stirring Time (hour)

Figure S5. Elasticity vs. stirring time for all investigated samples in (a) PANI-SBu-1 (b) PANI-SBu-2. The data points indicated by the red-dashed boxes are those discussed in the manuscript. The film elasticity initially increases with the stirring time and exhibits the largest value at the optimal stirring time, that is, 162 and 143 hours, respectively for PANI-SBu-1 and PANI-SBu-2. Beyond the optimal stirring time, the film elasticity decreases and eventually approaches a constant value. 
5. Multiple peak fitting for the elasticity distribution of PANI-SBu-2-179:

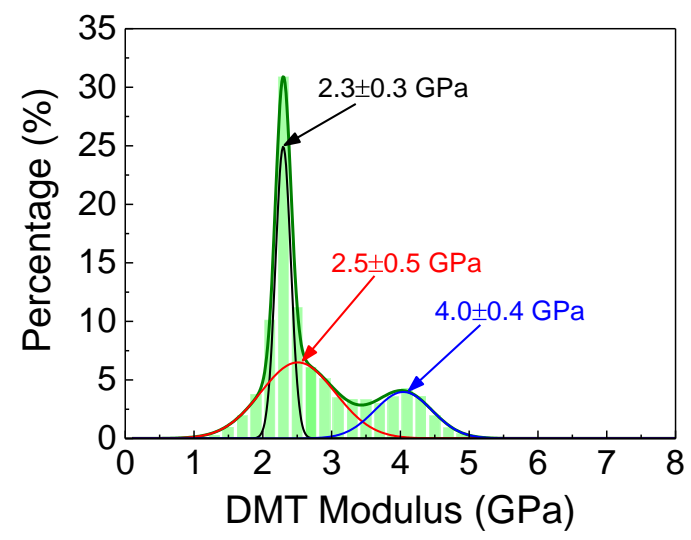

Figure S6. Because the elasticity distribution of elasticity values of PANI-SBu-2-179 is not a standard random distribution, we fit the data by using three Gaussian functions. The peak values of the obtained three Gaussian functions are respectively $2.3 \pm 0.3 \mathrm{GPa}, 2.5 \pm 0.5 \mathrm{GPa}$, and 4.0 $\pm 0.4 \mathrm{GPa}$. The olive solidline is the cumulative fit peak of the data.

7. The measured film roughness $R_{\mathrm{q}}$, elasticity $E$, film thickness $t$, mobility $\mu$, disorder and parameter $\gamma$ vs. solution stirring time for PANI-SBu-2.

Table S1. Summary of the obtained parameters for films in PANI-SBu-2.

\begin{tabular}{ccccc} 
Time (hours) & $\mathbf{5 0}$ & $\mathbf{9 1}$ & $\mathbf{1 4 3}$ & $\mathbf{1 7 9}$ \\
$\mathbf{E}(\mathbf{G P a})$ & $1.4 \pm 0.4$ & $2.4 \pm 0.4$ & $3.4 \pm 1.4$ & $2.3 \pm 0.1$ \\
$\boldsymbol{R}_{\mathbf{q}}(\mathbf{n m})$ & $11.3 \pm 2.6$ & $9.0 \pm 0.8$ & $5.3 \pm 0.6$ & $8.7 \pm 0.6$ \\
$\boldsymbol{t}$ on Si (nm) & $1247.7 \pm 43.8$ & $602.9 \pm 8.2$ & $1058 \pm 10.0$ & $491.0 \pm 16.0$ \\
$\boldsymbol{t}$ on ITO (nm) & $133.0 \pm 27.0$ & $393.0 \pm 13.2$ & $712.4 \pm 12.5$ & $157.5 \pm 19.7$ \\
$\boldsymbol{\mu}\left(\mathbf{c m}^{\mathbf{2}} \mathbf{V}^{-\mathbf{1}} \mathbf{s}^{-\mathbf{1}}\right)$ & $(1.8 \pm 0.6) \times 10^{-4}$ & $(4.0 \pm 0.5) \times 10^{-4}$ & $(5.2 \pm 0.1) \times 10^{-4}$ & $(4.7 \pm 0.3) \times 10^{-5}$ \\
$\boldsymbol{\gamma}\left(\mathbf{m}^{\mathbf{1} / \mathbf{2}} / \mathbf{V}^{\mathbf{1} / \mathbf{2}}\right)$ & $(5.8 \pm 0.5) \times 10^{-9}$ & $(-2.7 \pm 0.1) \times 10^{-8}$ & $(-4.9 \pm 0.2) \times 10^{-8}$ & $(-2.3 \pm 0.1) \times 10^{-8}$ \\
\hline
\end{tabular}


8. AFM and SEM images of PANI-SBu-2:
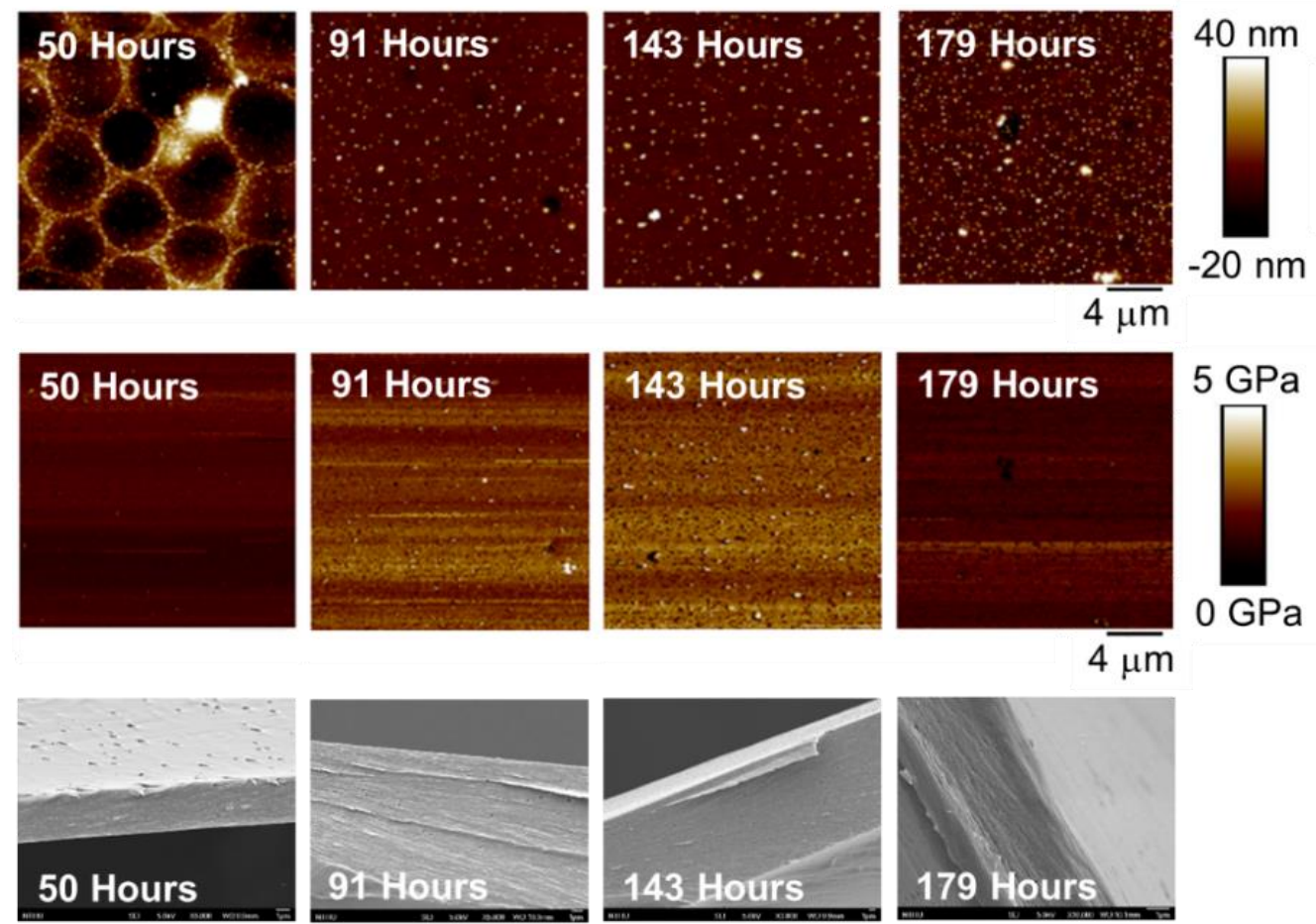

Figure S7. The AFM topography images, elastic moduli mappings, and the SEM images of the film cross-section area of PANI-SBu-2 thin films.

\section{Raw I-V curves of PANI-SBu-1 and PANI-SBu-2:}
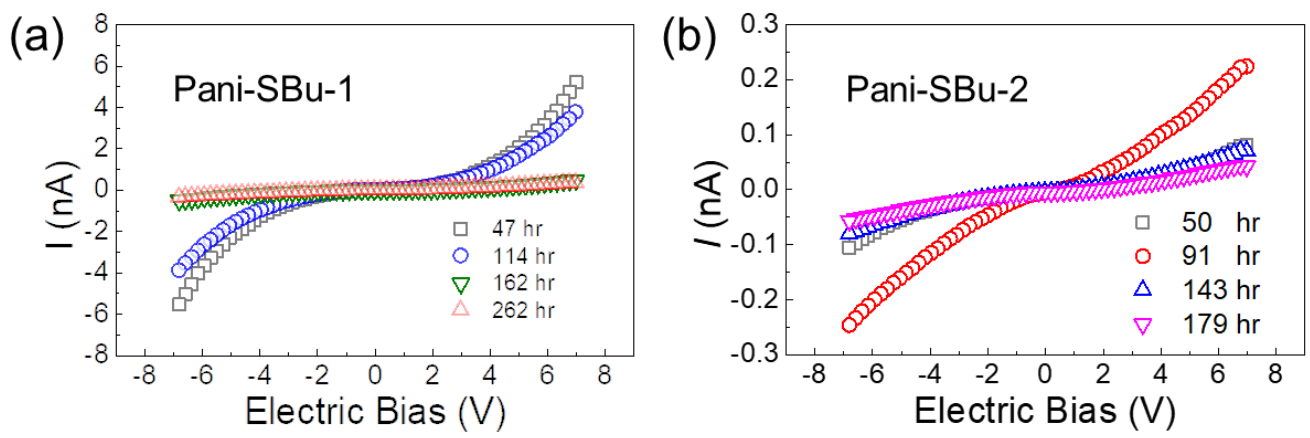

Figure S8. The raw $I V$ curves measured by cAFM on PANI-SBu-1, and -2, are shown in (a) and(b), respectively.

\section{Reference:}

(1) Lu, F. L.; Wudl, F.; Nowak, M.; Heeger, A. J., Phenyl-capped octaaniline (COA): an excellent model for polyaniline. Journal of the American Chemical Society 1986, 108 (26), 8311-8313.

(2) Epstein, A. J.; Ginder, J. M.; Zuo, F.; Bigelow, R. W.; Woo, H. S.; Tanner, D. B.; Richter, A. F.; Huang, W.

S.; MacDiarmid, A. G., Insulator-to-metal transition in polyaniline. Synthetic Metals 1987, 18 (1), 303-309.

(3) Han, C.-C.; Jeng, R.-C., Concurrent reduction and modification of polyaniline emeraldine base with pyrrolidine and other nucleophiles. Chemical Communications 1997, (6), 553-554. 
(4) Han, C.-C.; Hseih, W.-D.; Yeh, J.-Y.; Hong, S.-P., Combination of Electrochemistry with Concurrent Reduction and Substitution Chemistry To Provide a Facile and Versatile Tool for Preparing Highly

Functionalized Polyanilines. Chemistry of Materials 1999, 11 (2), 480-486.

(5) Han, C.-C.; Hong, S.-P.; Yang, K.-F.; Bai, M.-Y.; Lu, C.-H.; Huang, C.-S., Highly Conductive New Aniline Copolymers Containing Butylthio Substituent. Macromolecules 2001, 34 (3), 587-591.

(6) Han, C.-C.; Lu, C.-H.; Hong, S.-P.; Yang, K.-F., Highly Conductive and Thermally Stable Self-doping Propylthiosulfonated Polyanilines. Macromolecules 2003, 36 (21), 7908-7915.

(7) Han, C.-C.; Yang, K.-F.; Hong, S.-P.; Balasubramanian, A.; Lee, Y.-T., Syntheses and characterizations of aniline/butylthioaniline copolymers: Comparisons of copolymers prepared by the new concurrent reduction and substitution route and the conventional oxidative copolymerization method. Journal of Polymer Science Part A: Polymer Chemistry 2005, 43 (9), 1767-1777.

(8) Han, C.-C.; Chen, H.-Y., Highly Conductive and Electroactive Fluorine-Functionalized Polyanilines. Macromolecules 2007, 40 (25), 8969-8973.

(9) Han, C.-C.; Balakumar, R.; Thirumalai, D.; Chung, M.-T., The different electronic natures displayed by the alkylthio groups in simple and higher conjugated aniline systems. Organic \& Biomolecular Chemistry 2006, 4 (18), 3511-3516. 